PROCEEDINGS OF THE

AMERICAN MATHEMATICAL SOCIETY

Volume 140, Number 6, June 2012, Pages 1909-1919

S 0002-9939(2011)11052-9

Article electronically published on October 12, 2011

\title{
PATH COUNT ASYMPTOTICS AND STIRLING NUMBERS
}

\author{
K. PETERSEN AND A. VARCHENKO \\ (Communicated by Bernd Ulrich)
}

\begin{abstract}
We obtain formulas for the growth rate of the numbers of certain paths in a multi-dimensional analogue of the Eulerian graph. Corollaries are new identities relating Stirling numbers of the first and second kinds.
\end{abstract}

\section{INTRODUCTION}

A graded, infinite, directed graph defines a dynamical system called a BratteliVershik or adic system (see [8] and the references given there for background on adic systems). One of the main problems concerning these systems is to determine their ergodic invariant measures, and the basic tool for attacking this problem is the asymptotics of numbers of paths in the graph with certain properties.

A very interesting Bratteli-Vershik dynamical system is associated to the Eulerian two-dimensional graph with vertices at the points of $\mathbb{Z}_{>0}^{2}$. The paths in the Eulerian graph may be counted in many different combinatorial ways, but all attempts to extend those counts to the multi-dimensional analogues of the Eulerian graph have failed up to this moment; techniques applied to the two-dimensional Eulerian graph, such as the recurrence of random walks in two dimensions in [1, the coding of paths by permutations in [5], or the left-to-right monotonicity in [7, are not available in dimensions higher than two.

In this paper we make a first step in the multi-dimensional direction. We consider a multi-dimensional analogue of the Eulerian graph and determine the asymptotics of the numbers of paths with certain properties. We plan to apply these asymptotics to studying the ergodic measures on these and related systems in future work. As a rather unexpected corollary of our asymptotics we get a family of new identities between Stirling numbers of the first and second kinds, which are classical objects, arise in a variety of combinatorics problems, and for which many general relations have long been known (see for example [9, 10]).

Now we formulate the results of this paper. Denote by $e_{1}=(1,0, \ldots, 0), \ldots$, $e_{n+1}=(0, \ldots, 0,1) \in \mathbb{Z}^{n+1}$ the ordinary basis vectors in $\mathbb{R}^{n+1}$. Let $c \in \mathbb{Z}_{>0}^{n+1}$. We define the graph $E_{c}$ to be the graph with vertices $i \in \mathbb{Z}_{\geq 0}^{n+1}$ and the following directed multiple edges. Each vertex $i$ is connected to just $n+1$ vertices: to vertex

Received by the editors December 8, 2009 and, in revised form, February 7, 2011.

2010 Mathematics Subject Classification. Primary 05A10, 05A16, 05A19, 05C30, 05C63; Secondary 37A05, 37A50.

Key words and phrases. Eulerian numbers, Stirling numbers, symmetric polynomials, reinforced random walks, urn models.

The research of the second author was supported in part by NSF grant DMS-0555327.

(C)2011 American Mathematical Society 
$i+e_{j}$ by $c_{j}+i_{n+1}$ edges if $j<n+1$ and to vertex $i+e_{n+1}$ by $c_{n+1}+i_{1}+i_{2}+\cdots+i_{n}$ edges.

Such a graph models a random walk on the graph $G_{n+1}$ consisting of $n+1$ loops based at a single vertex, with a special kind of opposite reinforcement: when the walker takes a step in the direction of $e_{n+1}$, the numbers of edges in all the other directions $\left(e_{j}\right.$ for $\left.j=1, \ldots, n\right)$ are incremented by 1 , but when the walker takes a step in the direction of $e_{j}$ for some $j=1, \ldots, n$, only the number of edges in the direction of $e_{n+1}$ is incremented by 1 (cf. [6]). When $n=1$ and $c=(1,1), E_{c}$ is the standard two-dimensional Eulerian graph. The corresponding two-dimensional Eulerian adic system was introduced in [1] and studied further in [5] and 8; see also 2,4,7,

For each $i \in \mathbb{Z}_{>0}^{n+1}$ denote by $A_{c}(i)$ the number of paths in the graph $E_{c}$ from 0 to $i$. The standard Eulerian numbers (see 3,8 ) are the numbers $A\left(i_{1}, i_{2}\right)=$ $A_{(0,0)}\left(i_{1}, i_{2}\right)$ with $n=1$. In this paper, we give a formula for the leading term of the growth rate of $A_{c}\left(i_{1}, \ldots, i_{n}, i_{n+1}\right)$ as $i_{n+1}$ tends to infinity. We show that if $m=i_{1}+\cdots+i_{n}$, then

$$
A_{c}\left(i_{1}, \ldots, i_{n}, i_{n+1}\right) \sim B_{c}\left(i_{1}, \ldots, i_{n}\right)\left(c_{n+1}+m\right)^{i_{n+1}}
$$

as $i_{n+1} \rightarrow \infty$ and

$$
B_{c_{1}, \ldots, c_{n+1}}\left(i_{1}, \ldots, i_{n}\right)=\frac{\left(c_{n+1}+m\right)^{m}}{m !} \sum_{f \in F} \prod_{j=1}^{m}\left(c_{f(j)}+c_{n+1}+j-1\right),
$$

where $F$ denotes the set of all maps $f:\{1, \ldots, m\} \rightarrow\{1, \ldots, n\}$ such that $\left|f^{-1}(j)\right|=$ $i_{j}$ for $j=1, \ldots, n$.

As a corollary we obtain new identities relating Stirling numbers of the first and second kinds; for $1 \leq k \leq n, 0 \leq r \leq k$, we get

$$
\begin{gathered}
\left(\begin{array}{c}
r+n-k-1 \\
r
\end{array}\right) s_{1}(n, r+n-k) \\
=\sum_{m=0}^{k}\left(\begin{array}{c}
m+n-k \\
m+1
\end{array}\right) \sum_{i=0}^{r}\left(\begin{array}{c}
i+n-k+m-1 \\
i
\end{array}\right) \frac{(-1)^{m+r-i}}{n^{r-i+1}}(r-i+1) ! \\
\times s_{2}(m+1, r-i+1) s_{1}(n, i+n-k+m),
\end{gathered}
$$

where $s_{1}, s_{2}$ are Stirling numbers of the first and second kinds; see Theorem 3.10 and Corollary 3.11 .

\section{Path COUnts AND Limits}

2.1. Finding the leading term. The numbers $A_{c}(i)$ of paths in the graph $E_{c}$ from 0 to $i$ satisfy the recurrence relation

$$
A_{c}(i)=\left(c_{n+1}+i_{1}+\cdots+i_{n}\right) A_{c}\left(i-e_{n+1}\right)+\sum_{j=1}^{n}\left(c_{j}+i_{n+1}\right) A_{c}\left(i-e_{j}\right) .
$$

We are interested in the limit

$$
B_{c}\left(i_{1}, \ldots, i_{n}\right)=\lim _{i_{n+1} \rightarrow \infty} \frac{A_{c}\left(i_{1}, \ldots, i_{n}, i_{n+1}\right)}{\left(c_{n+1}+n\right)^{i_{n+1}}} .
$$


Proposition 2.1. Let $u=(1, \ldots, 1) \in \mathbb{Z}_{\geq 0}^{n}$. Then

$$
B_{c}(u)=\sum_{\xi \in S_{n}} \sum_{j_{1}, \ldots, j_{n}=0}^{\infty} \prod_{k=1}^{n}\left(c_{\xi(k)}+j_{1}+\cdots+j_{k}\right)\left(\frac{c_{n+1}+k-1}{c_{n+1}+n}\right)^{j_{k}} .
$$

Proof. To form a path from 0 to $i=\left(1, \ldots, 1, i_{n+1}\right)$ in $E_{c}$, we need to take one step in the direction of each $e_{k}, k=1, \ldots, n$, and $i_{n+1}$ steps in the direction of $e_{n+1}$. Thus each such path will correspond to a permutation $\xi$ of $1,2, \ldots, n$ which will determine when the step in the direction of each $e_{k}, k=1, \ldots, n$, is taken, together with a partition $i_{n+1}=j_{1}+\cdots+j_{n}+j_{n+1}$ of $i_{n+1}$ into nonnegative integers, which will determine how many consecutive steps are taken in the $e_{n+1}$ direction between steps in other directions. Each time that $j$ steps are taken in the $e_{n+1}$ direction, all the $c_{k}, 1 \leq k \leq n$, are incremented by 1 , and each time that a step is taken in a direction other than $e_{n+1}, c_{n+1}$ is incremented by 1 . Therefore,

$$
\begin{aligned}
& A_{c}\left(1, \ldots, 1, i_{n+1}\right) \\
& =\sum_{\xi \in S_{n}} \sum_{j_{1}+\cdots+j_{n+1}=i_{n+1}} c_{n+1}^{j_{1}}\left(c_{\xi(1)}+j_{1}\right)\left(c_{n+1}+1\right)^{j_{2}}\left(c_{\xi(2)}+j_{1}+j_{2}\right) \cdots \\
& \quad \cdots\left(c_{\xi(n)}+j_{1}+\cdots+j_{n}\right)\left(c_{n+1}+n\right)^{j_{n+1}} \\
& =\sum_{\xi \in S_{n}} \sum_{j_{1}+\cdots+j_{n+1}=i_{n+1}}\left[\prod_{k=1}^{n}\left(c_{n+1}+k-1\right)^{j_{k}}\left(c_{\xi(k)}+j_{1}+\cdots+j_{k}\right)\right]\left(c_{n+1}+n\right)^{j_{n+1}} \\
& =\left(c_{n+1}+n\right)^{i_{n+1}} \sum_{\xi \in S_{n}} \sum_{j_{1}+\cdots+j_{n+1}=i_{n+1}} \prod_{k=1}^{n}\left[\frac{\left(c_{n+1}+k-1\right)}{c_{n+1}+n}\right]^{j_{k}}\left(c_{\xi(k)}+j_{1}+\cdots+j_{k}\right) \\
& =\left(c_{n+1}+n\right)^{i_{n+1}} \sum_{\xi \in S_{n}} \sum_{\substack{j_{s} \geq 0 \\
j_{1}+\cdots+j_{n}=0}}^{n} \prod_{k=1}^{i_{n+1}}\left[\frac{\left(c_{n+1}+k-1\right)}{c_{n+1}+n}\right]^{j_{k}}\left(c_{\xi(k)}+j_{1}+\cdots+j_{k}\right) .
\end{aligned}
$$

This reasoning gives Formula (2.3). Clearly, $B_{c}(u)$ is a convergent sum.

Similarly, existence of the limit in Formula (2.2) for any $\left(i_{1}, \ldots, i_{n}\right)$ can be shown as follows. Let $m, F$ be the same as in Section 1. Then

$$
\begin{aligned}
& A_{c}\left(i_{1}, \ldots, i_{n}, i_{n+1}\right) \\
& =\sum_{f \in F} \sum_{j_{1}+\cdots+j_{m+1}=i_{n+1}} c_{n+1}^{j_{1}}\left(c_{f(1)}+j_{1}\right)\left(c_{n+1}+1\right)^{j_{2}}\left(c_{f(2)}+j_{1}+j_{2}\right) \cdots \\
& \quad \cdots\left(c_{f(m)}+j_{1}+\cdots+j_{m}\right)\left(c_{n+1}+m\right)^{j_{m+1}} \\
& =\left(c_{n+1}+m\right)^{i_{n+1}} \sum_{f \in F} \sum_{\substack{j_{s} \geq 0 \\
j_{1}+\cdots+j_{m}=0}}^{i_{n+1}} \prod_{k=1}^{m}\left[\frac{\left(c_{n+1}+k-1\right)}{c_{n+1}+m}\right]^{j_{k}}\left(c_{f(k)}+j_{1}+\cdots+j_{k}\right) .
\end{aligned}
$$

Dividing by $\left(c_{n+1}+m\right)^{i_{n+1}}$ and letting $i_{n+1} \rightarrow \infty$ again leaves a sum of convergent series. Thus we get

$$
B_{c}\left(i_{1}, \ldots, i_{n}\right)=\sum_{f \in F} \sum_{j_{1}, \ldots, j_{n}=0}^{\infty} \prod_{k=1}^{n}\left(c_{f(k)}+j_{1}+\cdots+j_{k}\right)\left(\frac{c_{n+1}+k-1}{c_{n+1}+n}\right)^{j_{k}}
$$


We shall give two formulas for $B_{c}(u)$. In this section we evaluate $B_{c}(u)$ using a two-dimensional formula from [8]. In Section 3.1 another formula is obtained by using derivatives of geometric series. Comparing the two formulas we get new identities involving Stirling numbers of the first and second kinds (Corollary 3.11).

Theorem 2.2. We have

$$
B_{c}(u)=\frac{\left(c_{n+1}+n\right)^{n}}{n !} \sum_{\xi \in S_{n}} \prod_{k=1}^{n}\left(c_{\xi(k)}+c_{n+1}+k-1\right) .
$$

2.2. Proof of Theorem 2.2. Denote by $\sigma_{i}(c), i=1, \ldots, n$, the elementary symmetric functions in $c$,

$$
\sigma_{i}(c)=\sum_{1 \leq j_{1}<\cdots<j_{i} \leq n} c_{j_{1}} \ldots c_{j_{i}} .
$$

Denote $\sigma_{0}(c)=1$. Clearly, the function $B_{c}(u)$ is a symmetric polynomial in $c$ of the form

$$
\begin{aligned}
& B_{c}(u)=\sum_{i=0}^{n} i !(n-i) ! \sigma_{i}(c) \alpha_{i, n}\left(c_{n+1}\right)=n ! \sigma_{n}(c) \alpha_{n, n}\left(c_{n+1}\right) \\
& +(n-1) ! \sigma_{n-1}(c) \alpha_{n-1, n}\left(c_{n+1}\right)+\cdots+n ! \sigma_{0}(c) \alpha_{0, n}\left(c_{n+1}\right),
\end{aligned}
$$

where $\alpha_{i, n}\left(c_{n+1}\right), i=0, \ldots, n$, are suitable functions in $c_{n+1}$. For example,

$$
\begin{aligned}
& \alpha_{0, n}\left(c_{n+1}\right)=\sum_{j_{1}, \ldots, j_{n}=0}^{\infty} \prod_{k=1}^{n}\left(j_{1}+\cdots+j_{k}\right)\left(\frac{c_{n+1}+k-1}{c_{n+1}+n}\right)^{j_{k}}, \\
& \alpha_{n, n}\left(c_{n+1}\right)=\sum_{j_{1}, \ldots, j_{n}=0}^{\infty} \prod_{k=1}^{n}\left(\frac{c_{n+1}+k-1}{c_{n+1}+n}\right)^{j_{k}}
\end{aligned}
$$

and so on.

Example 2.3. For $n=3$,

$$
\begin{aligned}
B_{\left(c_{1}, c_{2}, c_{3}\right)}(u)= & \sum_{j_{1}, j_{2}=0}^{\infty}\left[\left(c_{1}+j_{1}\right)\left(c_{2}+j_{1}+j_{2}\right)+\left(c_{2}+j_{1}\right)\left(c_{1}+j_{1}+j_{2}\right)\right] \\
& \times\left(\frac{c_{3}}{c_{3}+2}\right)^{j_{1}}\left(\frac{c_{3}+1}{c_{3}+2}\right)^{j_{2}} \\
= & 2 ! c_{1} c_{2} \alpha_{2,2}\left(c_{3}\right)+\left(c_{1}+c_{2}\right) \alpha_{1,2}\left(c_{3}\right)+2 ! \alpha_{0,2}\left(c_{3}\right),
\end{aligned}
$$

where

$$
\begin{aligned}
& \alpha_{2,2}\left(c_{3}\right)=\sum_{j_{1}, j_{2}=0}^{\infty}\left(\frac{c_{3}}{c_{3}+2}\right)^{j_{1}}\left(\frac{c_{3}+1}{c_{3}+2}\right)^{j_{2}}, \\
& \alpha_{1,2}\left(c_{3}\right)=\sum_{j_{1}, j_{2}=0}^{\infty}\left(2 j_{1}+j_{2}\right)\left(\frac{c_{3}}{c_{3}+2}\right)^{j_{1}}\left(\frac{c_{3}+1}{c_{3}+2}\right)^{j_{2}}, \\
& \alpha_{0,2}\left(c_{3}\right)=\sum_{j_{1}, j_{2}=0}^{\infty} j_{1}\left(j_{1}+j_{2}\right)\left(\frac{c_{3}}{c_{3}+2}\right)^{j_{1}}\left(\frac{c_{3}+1}{c_{3}+2}\right)^{j_{2}} .
\end{aligned}
$$


Our goal is to calculate all the coefficients $\alpha_{i, n}\left(c_{n+1}\right)$ and show that the result gives Formula (2.7). To do so let us calculate the number

$$
B_{c_{1}, c_{2}}(n)=\sum_{j_{1}, \ldots, j_{n}=0}^{\infty} \prod_{k=1}^{n}\left(c_{1}+j_{1}+\cdots+j_{k}\right)\left(\frac{c_{2}+k-1}{c_{2}+n}\right)^{j_{k}} .
$$

The function $B_{c_{1}, c_{2}}(n)$ has the form

$$
B_{c_{1}, c_{2}}(n)=c_{1}^{n} \alpha_{n, n}\left(c_{2}\right)+c_{1}^{n-1} \alpha_{n-1, n}\left(c_{2}\right)+\cdots+\alpha_{0, n}\left(c_{2}\right)
$$

where the functions $\alpha_{i, n}, i=0, \ldots, n$, are the same functions of one variable as in (2.9). (To see this, note the identical form of the two products in formulas (2.3) and (2.13), and note that the sum over the symmetric group is absorbed into the coefficients that do not involve $c_{n+1}$.)

Example 2.4. We have

$$
\begin{aligned}
B_{c_{1}, c_{2}}(2) & =\sum_{j_{1}, j_{2}=0}^{\infty}\left(c_{1}+j_{1}\right)\left(c_{1}+j_{1}+j_{2}\right)\left(\frac{c_{2}}{c_{2}+2}\right)^{j_{1}}\left(\frac{c_{2}+1}{c_{2}+2}\right)^{j_{2}} \\
& =c_{1}^{2} \alpha_{2,2}\left(c_{2}\right)+c_{1} \alpha_{1,2}\left(c_{2}\right)+\alpha_{0,2}\left(c_{2}\right)
\end{aligned}
$$

where the functions $\alpha_{0,2}, \alpha_{1,2}, \alpha_{2,2}$ are the same functions of one variable as in (2.12).

To calculate the functions $\alpha_{i, n}$ and prove Theorem 2.2 it is enough to calculate $B_{c_{1}, c_{2}}(n)$.

Lemma 2.5. We have

$$
B_{c_{1}, c_{2}}(n)=\frac{\left(c_{2}+n\right)^{n}}{n !} \prod_{k=1}^{n}\left(c_{1}+c_{2}+k-1\right) .
$$

Proof. Let us use formula (1.1) of [8] to evaluate

$$
B_{c_{1}, c_{2}}(n)=\lim _{i \rightarrow \infty} \frac{A_{c_{1}, c_{2}}(n, i)}{\left(c_{2}+n\right)^{i}} .
$$

Formula (1.1) for $A_{c_{1}, c_{2}}(n, i)$ has $n+1$ summands and the leading summand is

$$
\left(\begin{array}{c}
c_{1}+c_{2}+n-1 \\
n
\end{array}\right)\left(c_{2}+n\right)^{n+i}
$$

This term after division by $\left(c_{2}+n\right)^{i}$ gives the right hand side of (2.17). The lemma is proved.

Lemma 2.5 gives Theorem 2.2

2.3. Formula for $B_{c}(i)$. Formula (2.7) for $B_{c_{1}, \ldots, c_{n+1}}(1, \ldots, 1)$ easily gives a formula for $B_{c_{1}, \ldots, c_{n+1}}\left(i_{1}, \ldots, i_{n}\right)$ for any $\left(i_{1}, \ldots, i_{n}\right)$.

Proposition 2.6. Let $m, F$, be the same as in Section 1. Then

$$
B_{c_{1}, \ldots, c_{n+1}}\left(i_{1}, \ldots, i_{n}\right)=\frac{\left(c_{n+1}+m\right)^{m}}{m !} \sum_{f \in F} \prod_{j=1}^{m}\left(c_{f(j)}+c_{n+1}+j-1\right) .
$$

\section{STIRLING NUMBERS AND RELATED POLYNOMials}

Theorem 2.2 has as corollaries interesting identities involving Stirling numbers of the first and second kinds. 
3.1. Theorem 2.2 in new notation. Denote

$$
u_{a}=\frac{q-a}{q}, \quad D_{a}=u_{a} \frac{\partial}{\partial u_{a}} .
$$

Then Theorem 2.2 says that for arbitrary numbers $c_{1}, \ldots, c_{n}$ we have

$$
\left(\sum_{\sigma \in S_{n}} \prod_{a=1}^{n}\left(c_{\sigma(a)}+D_{a}+D_{a+1}+\cdots+D_{n}\right)\right) \prod_{b=1}^{n} \frac{1}{1-u_{b}}=\frac{q^{n}}{n !} \sum_{\sigma \in S_{n}} \prod_{a=1}^{n}\left(c_{\sigma(a)}+q-a\right) .
$$

Example 3.1. For $n=2$, Formula (3.1) says that

$$
\begin{aligned}
{\left[( c _ { 1 } + D _ { 2 } ) \left(c_{2}+D_{2}+\right.\right.} & \left.\left.D_{1}\right)+\left(c_{2}+D_{2}\right)\left(c_{1}+D_{2}+D_{1}\right)\right] \frac{1}{1-u_{1}} \frac{1}{1-u_{2}} \\
& =\frac{q^{2}}{2}\left[\left(c_{1}+q-1\right)\left(c_{2}+q-2\right)+\left(c_{2}+q-1\right)\left(c_{1}+q-2\right)\right] .
\end{aligned}
$$

This statement is equivalent to the identities

$$
\begin{aligned}
\frac{1}{1-u_{1}} \frac{1}{1-u_{2}} & =\frac{q^{2}}{2}, \\
\left(2 D_{2}+D_{1}\right) \frac{1}{1-u_{1}} \frac{1}{1-u_{2}} & =\frac{q^{2}}{2}(q-2+q-1), \\
D_{2}\left(D_{2}+D_{1}\right) \frac{1}{1-u_{1}} \frac{1}{1-u_{2}} & =\frac{q^{2}}{2}(q-1)(q-2) .
\end{aligned}
$$

3.2. Formula for $\left(u \partial_{u}\right)^{k}(1-u)^{-1}$. We make some observations that are useful for handling expressions such as the left side of Formula (3.1).

Let $q$ be a variable, $a$ a positive integer. Set

$$
u=\frac{q-a}{q}, \quad \partial_{u}=\frac{\partial}{\partial u} .
$$

Let $A(i, j)=A_{0,0}(i, j)$ be the standard Eulerian numbers as in Section 1 .

Theorem 3.2. For any positive integer $k$ we have

$$
\left(u \partial_{u}\right)^{k}(1-u)^{-1}=a^{-k-1} \sum_{i+j=k-1} A(i, j) q^{i+1}(q-a)^{j+1} .
$$

\section{Example 3.3.}

$$
\begin{aligned}
u \partial_{u}(1-u)^{-1} & =a^{-2} q(q-a), \\
\left(u \partial_{u}\right)^{2}(1-u)^{-1} & =a^{-3}\left(q^{2}(q-a)+q(q-a)^{2}\right), \\
\left(u \partial_{u}\right)^{3}(1-u)^{-1} & =a^{-4}\left(q^{3}(q-a)+4 q^{2}(q-a)^{2}+q(q-a)^{3}\right) .
\end{aligned}
$$

Theorem 3.2 follows by induction from the following lemma.

Lemma 3.4. We have

$$
u \frac{\partial}{\partial u}=\frac{q(q-a)}{a} \frac{\partial}{\partial q} .
$$


Proof of Theorem 3.2 .

$$
\begin{aligned}
& \frac{q(q-a)}{a} \frac{\partial}{\partial q} a^{-k-1} \sum_{i+j=k-1} A(i, j) q^{i+1}(q-a)^{j+1} \\
& =a^{-k-2} \sum_{i+j=k-1} A(i, j)(i+1) q^{i+1}(q-a)^{j+2} \\
& +a^{-k-2} \sum_{i+j=k-1} A(i, i)(j+1) q^{i+2}(q-a)^{j+1} \\
& =a^{-k-2} \sum_{r+s=k} A(r, s-1)(r+1) q^{r+1}(q-a)^{s+1} \\
& +a^{-k-2} \sum_{r+s=k} A(r-1, s)(s+1) q^{r+1}(q-a)^{s+1} \\
& =a^{-k-2} \sum_{r+s=k} A(r, s) q^{r+1}(q-a)^{s+1} .
\end{aligned}
$$

3.3. $\Gamma$-polynomials. We shall reformulate Theorem 3.2 in terms of a slight variation of Eulerian polynomials (see [3, p. 244]), which we call $\Gamma$-polynomials.

Let $s_{2}(k, m)$ denote Stirling numbers of the second kind, which count the number of partitions of a $k$-element set into $m$ nonempty subsets, so that

$$
\begin{gathered}
s_{2}(1,1)=1, \\
s_{2}(2,1)=1, \quad s_{2}(2,2)=1, \\
s_{2}(3,1)=1, \quad s_{2}(3,2)=3, \quad s_{2}(3,3)=1 .
\end{gathered}
$$

We have

$$
s_{2}(k, m)=s_{2}(k-1, m-1)+m s_{2}(k-1, m) .
$$

We will be interested in the polynomials

$$
\Gamma_{k}(q, n)=\sum_{i=1}^{k}(-1)^{k-i} s_{2}(k, i) i ! q^{i-1} n^{k-i} .
$$

\section{Example 3.5.}

$$
\Gamma_{1}(q, n)=1, \quad \Gamma_{2}(q, n)=2 q-n, \quad \Gamma_{3}(q, n)=6 q^{2}-6 q n+n^{2} .
$$

Theorem 3.6 (Frobenius, [3, p. 244]). We have

$$
\Gamma_{k}(q, n)=\sum_{i+j=k-1} A(i, j) q^{i}(q-n)^{j} .
$$

3.4. $\Delta$-polynomials. We will also need $\Delta$-polynomials.

Looking at the right side of Formula (3.1) suggests investigating related polynomials and Stirling numbers of the first kind.

Stirling numbers of the first kind are the coefficients in the expansion

$$
(q)_{n}=\sum_{k=0}^{n} s_{1}(n, k) q^{k}
$$

where $(q)_{n}$ is the falling factorial

$$
(q)_{n}=q(q-1)(q-2) \cdots(q-n+1) .
$$


We have

$$
s_{1}(n, k)=\sum_{1 \leq i_{1}<\cdots<i_{n-k} \leq n-1}(-1)^{n-k} i_{1} i_{2} \cdots i_{n-k} .
$$

For natural numbers $1 \leq k<n$, define a polynomial

$$
\Delta_{n, k}(q)=\sum_{1 \leq i_{1}<\cdots<i_{n-k} \leq n-1}\left(q-i_{1}\right)\left(q-i_{2}\right) \cdots\left(q-i_{n-k}\right) .
$$

Define $\Delta_{n, n}(q)=1$. We have $\Delta_{n, 1}(q)=(q-1) \ldots(q-n+1)$ and

$$
\Delta_{n, k}(q)=\sum_{m=0}^{n-k}\left(\begin{array}{c}
k-1+m \\
m
\end{array}\right) s_{1}(n, k+m) q^{m} .
$$

Example 3.7.

$$
\Delta_{2,2}(q)=1, \quad \Delta_{2,1}(q)=q-1,
$$

$$
\Delta_{3,3}(q)=1, \quad \Delta_{3,2}(q)=2 q-3, \quad \Delta_{3,1}(q)=q^{2}-3 q+2 .
$$

\subsection{Relation between $\Gamma$ and $\Delta$ polynomials.}

Example 3.8. We have the following identity:

$$
\left(\begin{array}{cc}
2^{-1}\left(\begin{array}{l}
2 \\
1
\end{array}\right) \Gamma_{1}(q, 2) & 0 \\
2^{-2}\left(\begin{array}{l}
2 \\
2
\end{array}\right) \Gamma_{2}(q, 2) & 2^{-1}\left(\begin{array}{l}
1 \\
1
\end{array}\right) \Gamma_{1}(q, 2)
\end{array}\right)\left(\begin{array}{c}
\Delta_{2,2}(q) \\
\Delta_{2,1}(q)
\end{array}\right)=\left(\begin{array}{c}
\Delta_{2,2}(q) \\
\Delta_{2,1}(q)
\end{array}\right) .
$$

In other words, we have

$$
\begin{gathered}
\left(\begin{array}{l}
2 \\
1
\end{array}\right) \frac{\Gamma_{1}(q, 2) \Delta_{2,2}(q)}{2}=\Delta_{2,2}(q) \\
\left(\begin{array}{l}
2 \\
2
\end{array}\right) \frac{\Gamma_{2}(q, 2) \Delta_{2,2}(q)}{2^{2}}+\left(\begin{array}{l}
1 \\
1
\end{array}\right) \frac{\Gamma_{1}(q, 2) \Delta_{2,1}(q)}{2}=\Delta_{2,1}(q) .
\end{gathered}
$$

Example 3.9. We have the following identity:

$$
\left(\begin{array}{ccc}
3^{-1}\left(\begin{array}{l}
3 \\
1
\end{array}\right) \Gamma_{1}(q, 3) & 0 & 0 \\
3^{-2}\left(\begin{array}{l}
3 \\
2
\end{array}\right) \Gamma_{2}(q, 3) & 3^{-1}\left(\begin{array}{l}
2 \\
1
\end{array}\right) \Gamma_{1}(q, 3) & 0 \\
3^{-3}\left(\begin{array}{l}
3 \\
3
\end{array}\right) \Gamma_{3}(q, 3) & 3^{-2}\left(\begin{array}{l}
2 \\
2
\end{array}\right) \Gamma_{2}(q, 3) & 3^{-1}\left(\begin{array}{l}
1 \\
1
\end{array}\right) \Gamma_{1}(q, 3)
\end{array}\right)\left(\begin{array}{c}
\Delta_{3,3}(q) \\
\Delta_{3,2}(q) \\
\Delta_{3,1}(q)
\end{array}\right)=\left(\begin{array}{c}
\Delta_{3,3}(q) \\
\Delta_{3,2}(q) \\
\Delta_{3,1}(q)
\end{array}\right) .
$$

In other words, we have

$$
\begin{gathered}
\left(\begin{array}{l}
3 \\
1
\end{array}\right) \frac{\Gamma_{1}(q, 3) \Delta_{3,3}(q)}{3}=\Delta_{3,3}(q) \\
\left(\begin{array}{l}
3 \\
2
\end{array}\right) \frac{\Gamma_{2}(q, 3) \Delta_{3,3}(q)}{3^{2}}+\left(\begin{array}{l}
2 \\
1
\end{array}\right) \frac{\Gamma_{1}(q, 3) \Delta_{3,2}(q)}{3}=\Delta_{3,2}(q), \\
\left(\begin{array}{l}
3 \\
3
\end{array}\right) \frac{\Gamma_{3}(q, 3) \Delta_{3,3}(q)}{3^{3}}+\left(\begin{array}{l}
2 \\
2
\end{array}\right) \frac{\Gamma_{2}(q, 3) \Delta_{3,2}(q)}{3^{2}}+\left(\begin{array}{l}
1 \\
1
\end{array}\right) \frac{\Gamma_{1}(q, 3) \Delta_{3,1}(q)}{3}=\Delta_{3,1}(q) .
\end{gathered}
$$

More generally, we have the following relation.

Theorem 3.10. For any $1 \leq k \leq n$ we have

$$
\Delta_{n, k}(q)=\sum_{m=0}^{n-k}\left(\begin{array}{c}
k+m \\
1+m
\end{array}\right) \frac{\Gamma_{1+m}(q, n) \Delta_{n, k+m}(q)}{n^{1+m}} .
$$


Proof. Recall the notations $u_{b}$ and $D_{b}$ defined at the beginning of Section 3.1. Denote

$$
\pi_{n}=\prod_{b=1}^{n} \frac{1}{1-u_{b}}, \quad I_{n}^{k}(q)=\sum_{1 \leq i_{1}<\cdots<i_{k} \leq n}\left(\prod_{j=1}^{k}\left(D_{i_{j}}+D_{i_{j}+1}+\cdots+D_{n}\right)\right) \pi_{n} .
$$

For example,

$$
I_{n}^{1}(q)=\left(n D_{n}+(n-1) D_{n-1}+\cdots+D_{1}\right) \pi_{n} .
$$

Denote by $\sigma_{i}(c), i=1, \ldots, n$, the elementary symmetric functions in $c=\left(c_{1}, \ldots, c_{n}\right)$ as before.

The left hand side of Formula (3.1) equals

$$
\sum_{k=0}^{n} k !(n-k) ! \sigma_{n-k}(c) I_{n}^{k}(q)
$$

The right hand side equals

$$
\frac{q^{n}}{n !} \sum_{k=0}^{n} k !(n-k) ! \sigma_{n-k}(c) \Delta_{n+1, n-k+1}(q) .
$$

By comparing coefficients of the symmetric polynomials in these two equations we get

$$
I_{n}^{k}(q)=\frac{q^{n}}{n !} \Delta_{n+1, n-k+1}(q) .
$$

We have

$$
\Delta_{n+1, n-k+1}(q)-\Delta_{n, n-k}(q)=(q-n) \Delta_{n, n-k+1}(q)
$$

and

$$
I_{n}^{k}(q)=\sum_{j=0}^{k}\left(\begin{array}{l}
n-j \\
k-j
\end{array}\right) I_{n-1}^{j}(q)\left(\left(D_{n}\right)^{k-j} \frac{1}{1-u_{n}}\right) .
$$

Hence

$$
I_{n}^{k}(q)-I_{n-1}^{k}(q) \frac{1}{1-u_{n}}=\sum_{j=0}^{k-1}\left(\begin{array}{l}
n-j \\
k-j
\end{array}\right) I_{n-1}^{j}(q)\left(\left(D_{n}\right)^{k-j} \frac{1}{1-u_{n}}\right) .
$$

The left hand side of (3.7) is

$$
\begin{aligned}
I_{n}^{k}(q)-I_{n-1}^{k}(q) \frac{1}{1-u_{n}} & =I_{n}^{k}(q)-I_{n-1}^{k}(q) \frac{q}{n} \\
& =\frac{q^{n}}{n !} \Delta_{n+1, n-k+1}(q)-\frac{q^{n-1}}{(n-1) !} \Delta_{n, n-k}(q) \frac{q}{n} \\
& =\frac{q^{n}}{n !}\left(\Delta_{n+1, n-k+1}(q)-\Delta_{n, n-k}(q)\right) \\
& =\frac{q^{n}(q-n)}{n !} \Delta_{n, n-k+1}(q) .
\end{aligned}
$$


The right hand side of (3.7) equals

$$
\begin{gathered}
\sum_{j=0}^{k-1}\left(\begin{array}{l}
n-j \\
k-j
\end{array}\right) I_{n-1}^{j}(q)\left(\left(D_{n}\right)^{k-j} \frac{1}{1-u_{n}}\right) \\
=\sum_{j=0}^{k-1}\left(\begin{array}{l}
n-j \\
k-j
\end{array}\right) \frac{q^{n-1}}{(n-1) !} \Delta_{n, n-j}(q) \frac{q(q-n)}{n^{k-j+1}} \Gamma_{n-j}(q, n) .
\end{gathered}
$$

These two equations prove Theorem 3.10 .

Since $\Delta_{n, n}(q)=1$ and $\Gamma_{1}(q, n)=1$ for all $n$, identity (3.5) allows us to express the polynomials $\Delta_{n, k}(q)$ in terms of polynomials $\Gamma_{k}(q, n)$ and vice versa; see the examples above.

3.6. Identities involving Stirling numbers. The conclusion of Theorem 3.10 can be written in the following form:

$$
\Delta_{n, k}(q)=\frac{1}{n-k} \sum_{m=1}^{n-k}\left(\begin{array}{c}
m+k \\
m+1
\end{array}\right) \frac{1}{n^{m}} \Gamma_{m+1}(q, n) \Delta_{n, k+m}(q), \quad k=1, \ldots, n .
$$

Comparing coefficients of $q^{r}$ of the two sides yields identities involving the Stirling numbers $s_{1}(n, k)$ and $s_{2}(n, k)$ of the first and second kinds, indexed with $1 \leq k \leq n$ (and equaling 0 outside this range).

Corollary 3.11. For $1 \leq k \leq n, 0 \leq r \leq k$, we have

$$
\begin{gathered}
\left(\begin{array}{c}
r+n-k-1 \\
r
\end{array}\right) s_{1}(n, r+n-k) \\
=\sum_{m=0}^{k}\left(\begin{array}{c}
m+n-k \\
m+1
\end{array}\right) \sum_{i=0}^{r}\left(\begin{array}{c}
i+n-k+m-1 \\
i
\end{array}\right) \frac{(-1)^{m+r-i}}{n^{r-i+1}}(r-i+1) ! \\
\times s_{2}(m+1, r-i+1) s_{1}(n, i+n-k+m) .
\end{gathered}
$$

For $r=0$, this says that

$$
s_{1}(n, n-k)=\frac{n-k}{n} s_{1}(n, n-k)+\sum_{m=1}^{k}\left(\begin{array}{c}
m+n-k \\
m+1
\end{array}\right) \frac{(-1)^{m}}{n} s_{1}(n, n-k+m),
$$

and hence

$$
s_{1}(n, n-k)=\frac{1}{k} \sum_{m=1}^{k}\left(\begin{array}{c}
m+n-k \\
m+1
\end{array}\right)(-1)^{m} s_{1}(n, n-k+m), \quad k=1, \ldots, n .
$$

This contains the known formulas

$$
s_{1}(n, n-1)=-\left(\begin{array}{l}
n \\
2
\end{array}\right) \quad \text { and } \quad s_{1}(n, n-2)=\frac{1}{24} n(n-1)(n-2)(3 n-1) .
$$

\section{ACKNOWLEDGMENTS}

We thank R. Stanley for telling us about the theorem of Frobenius (Theorem 3.6) and the referee for suggestions leading to improvement of the exposition. 


\section{REFERENCES}

[1] S. Bailey, M. Keane, K. Petersen, and I. Salama, Ergodicity of the adic transformation on the Euler graph, Math. Proc. Camb. Phil. Soc. 141 (2006), no. 2, 231-238. MR2265871 (2007m:37010)

[2] L. Carlitz and R. Scoville, Generalized Eulerian numbers: combinatorial applications, J. Reine Angew. Math. 265 (1974), 110-137. MR0429575 (55:2587)

[3] L. Comtet, Advanced Combinatorics, The Art of Finite and Infinite Expansions, enlarged edition, D. Reidel Publishing Co., Dordrecht, 1974. MR0460128 (57:124)

[4] P. Flajolet, P. Dumas, and V. Puyhaubert, Some exactly solvable models of urn process theory, DMTCS Proceedings, Fourth Colloquium on Mathematics and Computer Science Algorithms, Trees, Combinatorics and Probabilities (2006), 59-118. http://www.dmtcs.org/ dmtcs-ojs/index.php/proceedings/issue/view/84.

[5] S. Frick and K. Petersen, Random permutations and unique fully supported ergodicity for the Euler adic transformation, Ann. Inst. Henri Poincaré Probab. Stat. 44 (2008), no. 5, 876-885 (English, with English and French summaries). MR2453848 (2009h:37004)

[6] _ Reinforced random walks and adic transformations, J. Theor. Prob. 23 (2010), 920-943. MR2679963

[7] A. Gnedin and G. Olshanski, The boundary of the Eulerian number triangle, Mosc. Math. J. 6 (2006), no. 3, 461-475. MR2274860(2007j:05006)

[8] K. Petersen and A. Varchenko, The Euler adic dynamical system and path counts in the Euler graph, Tokyo J. Math. 33 (2010), no. 2, 327-340. MR2779260

[9] N. Sloane, The On-Line Encyclopedia of Integer Sequences, http://oeis.org, 2010.

[10] N. Sloane and S. Plouffe, The Encyclopedia of Integer Sequences, Academic Press, Inc., San Diego, CA, 1995. With a separately available computer disk. MR1327059 (96a:11001)

Department of Mathematics, University of North Carolina at Chapel Hill, Chapel Hill, North Carolina 27599-3250

E-mail address: petersen@math.unc.edu

Department of Mathematics, University of North Carolina at Chapel Hill, Chapel Hill, North Carolina 27599-3250

E-mail address: anv@math.unc.edu 